\title{
MOUVEMENT CHAOTIQUE D'UN ENSEMBLE DE TOURBILLONS ET EMISSION ACOUSTIQUE ASSOCIÉE
}

L. COLLOREC et D. JUVE

Laboratoire de Mécanique des Fluides et d'Acoustique, CNRS URA-263, Ecole Centrale de Lyon, BP. 163, F.69131 Ecully cedex, France

\begin{abstract}
This paper describes a numerical simulation of the behaviour of three inviscid point vortices above a flat wall. Depending on their initial positions, the trajectories of the vortices exhibit patterns ranging from regular to chaotic. The chaotic motion of vortices results in high frequencies components in the wall pressure spectra. In addition, it increases the far field acoustic intensity.
\end{abstract}

\section{1 - INTRODUCTION}

Les phénomènes chaotiques des systèmes physiques ont fait l'objet de nombreuses études ces dernières années en mécanique des fluides mais aussi en acoustique. Il est donc tentant de chercher à faire le lien entre le caractère chaotique d'un écoulement et ses répercussions aussi bien sur la pression hydrodynamique que sur le champ acoustique lointain généré.

\section{2 - MODELE ADOPTE}

Aref [1] a montré que trois tourbillons placés près d'une paroi pouvaient présenter des comportements chaotiques ou non. C'est ce modèle que nous avons repris. Il s'agit de trois tourbillons rectilinéaires de même intensité dans un fluide irrotationnel bidimensionnel et placés près d'une paroi plane simulée par la méthode des images. Le comportement de ces tourbillons est traité par la méthode des potentiels complexes. Il est également possible de rajouter une vitesse de convection du fluide uniforme et parallèle à la paroi. Ce sont les positions initiales des tourbillons qui définissent leur comportement. L'étude paramétrique effectuée porte sur le paramètre $a$ défini figure 1 ;

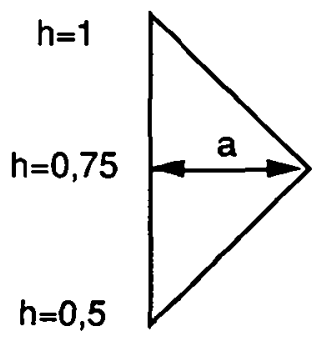

Figure I 
nous l'avons fait varier de $a=0.433$ (triangle équilatéral) à $a=0.00$ (triangle plat). Les trajectoires des tourbillons ont été calculées numériquement par une méthode de Runge-Kutta d'ordre 4.

Par ailleurs, la pression hydrodynamique en un point de la paroi a été calculée par la formule de Bernoulli $P=-\rho \frac{\partial \emptyset}{\partial t}-\rho \frac{V^{2}}{2}$ où $\varnothing$ est le potentiel des vitesses et $\rho$ la masse volumique du fluide. Enfin, le champ acoustique lointain est calculé à partir de la répartition de la vorticité $\vec{\omega}$ dans le fluide [2]. Nous supposons, pour simplifier le modèle, que le rayonnement se fait en trois dimensions.

Nous avons alors

$$
p=\frac{\rho}{4 \pi c_{0}^{2}} \frac{1}{|\vec{x}|}\left[\frac{d^{2}}{d t^{2}} \int(\vec{d} \cdot \vec{y})(\vec{d} \cdot \vec{L}) d y\right]^{*}
$$

où $\vec{x}$ désigne les coordonnées du point d'écoute et $\vec{d}=\frac{\vec{x}}{|\vec{x}|} ; \quad \vec{L}=-\vec{u} \wedge \vec{\omega}, \vec{u}$ est la vitesse du fluide et l'astérisque indique que l'expression entre crochets est évaluée au temps retardé $t^{*}=t-\frac{|\vec{x}|}{c_{0}} \quad\left(c_{0}\right.$ vitesse du son), la région source étant supposée compacte.

Compte tenu de la distribution discrète de vorticité et de la présence de tourbillons images, l'expression de la pression se ramène à :

$$
p=\frac{\rho 1}{2 c_{0}^{2}|\vec{x}|} \sum_{n=1}^{3} \frac{d^{2}}{{d t^{2}}^{2}}\left[\alpha_{n}\right]^{*} \quad \alpha_{n}=\frac{2 \gamma}{x^{2}+y^{2}}\left(x^{2} x_{n} v_{n}-y^{2} y_{n} u_{n}\right)
$$

où le tourbillon de position $\left(\begin{array}{l}x_{n} \\ y_{n}\end{array}\right)$ possède la vitesse $\left(\begin{array}{l}u_{n} \\ v_{n}\end{array}\right)$. I est la longueur des tourbillons filaments considérés. $\gamma$ est l'intensité tourbillonnaire.

Les simulations numériques montrent que les deux termes $\frac{d^{2}}{d t^{2}}\left[x_{n} v_{n}\right]^{*}$ et $\frac{d^{2}}{d t^{2}}\left[y_{n} u_{n}\right]^{*}$ sont toujours égaux, ce qui conduit à $p=\frac{\gamma \rho 1}{c_{0}^{2}|\vec{x}|} \frac{x^{2}-y^{2}}{x^{2}+y^{2}} \frac{d^{2}}{d t^{2}}\left[x_{n} v_{n}\right]^{*}$

La répartition de pression est donc quadripolaire.

\section{3 - RESULTATS OBTENUS}

\section{a - Trajectoires :}

Les figures 2 et 3 donnent les deux cas extrêmes observés. La figure 2 correspond au cas $a=0.433$, c'est-à-dire une conformation initiale des tourbillons en triangle équilatéral. Les trajectoires obtenues sont régulières et périodiques. La transformée de Fourier d'une coordonnée de trajectoire est composée de pics pointus régulièrement espacés : un fondamental et ses harmoniques. A mesure que l'on décroît la valeur du paramètre $a$, les pics observés dans la transformée de Fourier se déplacent 
vers les hautes fréquences tout en s'élargissant pour donner finalement un spectre de "large bande". Ceci se traduit dans l'espace physique par des trajectoires de tourbillons de plus en plus désordonnées ; ce comportement est illustré par la figure 3 pour le cas limite $a=0$.

3 UORTEX PRES DJUNE PAROI

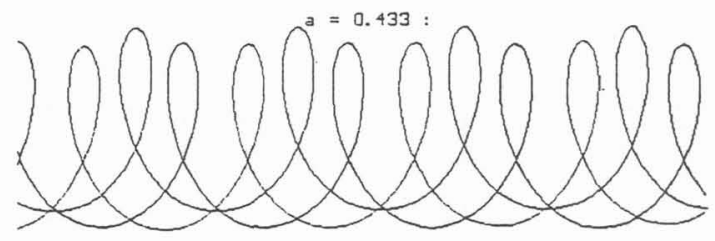

Figure 2
3 UORTEX PRES D'UNE PAROI

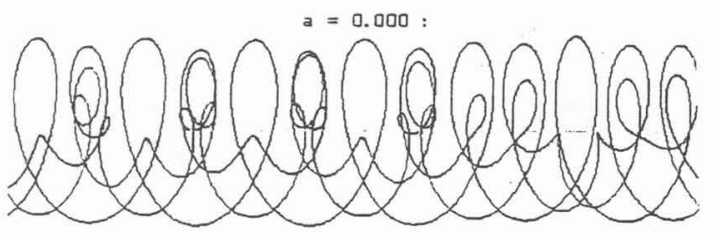

Figure 3

\section{b Pression à la paroi :}

Les figures 4 et 5 représentent la variation de la pression à la paroi lors du passage du groupe de tourbillons pour les deux cas extrêmes $a=0.433$ et $a=0$. La forme générale de la trace en pression est conservée et correspond à celle laissée par un seul tourbillon d'intensité égale à celle du groupe de tourbillons et placé à leur barycentre. Les oscillations de moindre amplitude superposées à la forme générale de la trace en pression proviennent de la rotation des tourbillons autour de leur barycentre. Plus leur mouvement est chaotique, plus l'amplitude et la fréquence de ces oscillations est grande.

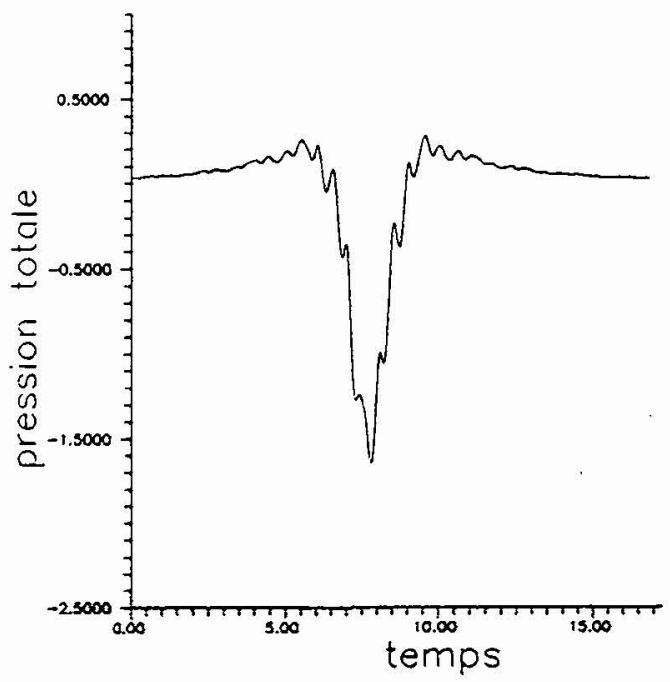

Figure 4

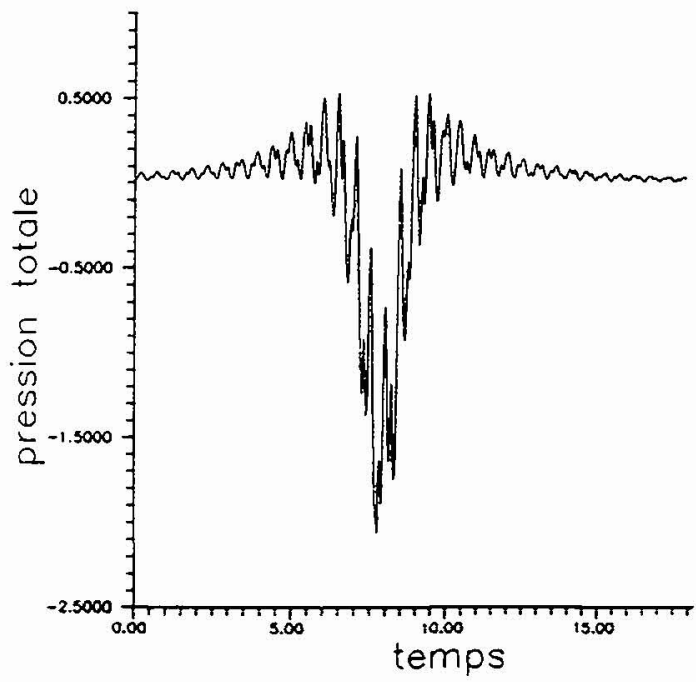

Figure 5 
c Champ acoustique lointain :

Comme nous l'avons dit précédemment la directivité du champ est quadripolaire

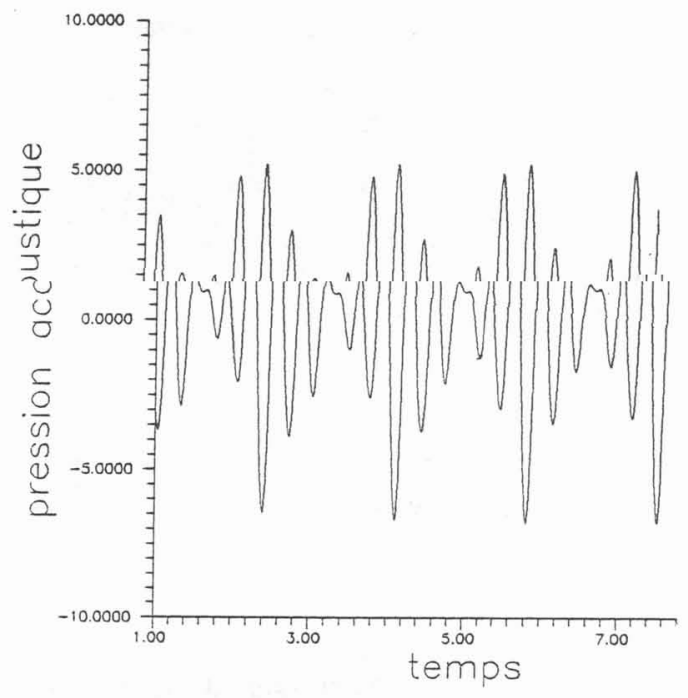

Figure 6

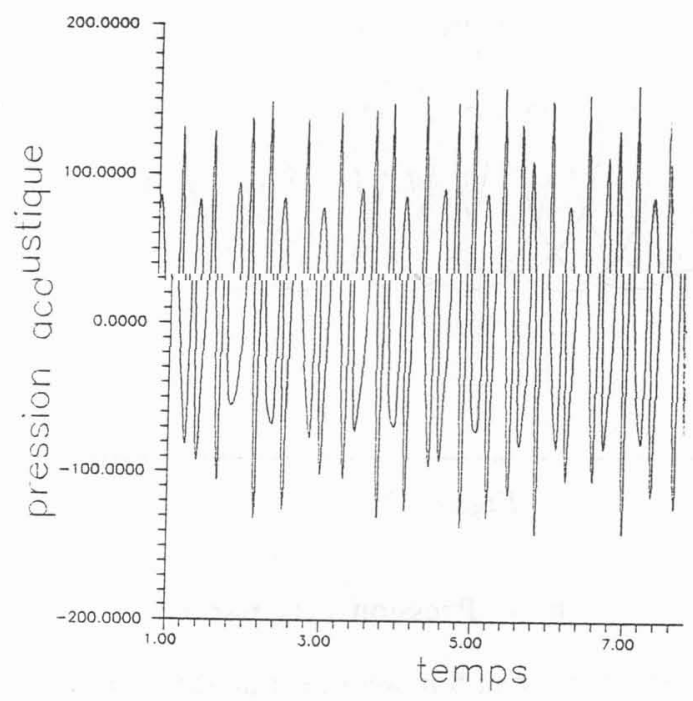

Figure 7

Les figures 6 et 7 montrent l'évolution au cours du temps de la pression acoustique (découplée du facteur de directivité). Dans le cas $a=0.433$ la pression évolue régulièrement même si une légère déformation apparaît au cours du temps. Au fur et à mesure que le paramètre $a$ diminue, la déformation s'accentue et l'amplitude des oscillations de pression augmente (noter la différence des échelles des figures 6 et 7). Cette augmentation est très forte puisqu'il y a un facteur 20 entre la configuration du triangle équilatéral et celle du triangle plat.

\section{4- CONCLUSIONS}

Cette étude fait le lien entre le comportement régulier ou chaotique d'un groupe de trois tourbillons près d'une paroi et la pression hydrodynamique ou acoustique qui en resulte. Plus le comportement des tourbillons est chaotique, plus la trace en pression à la paroi présente de hautes fréquences. Le trait marquant concernant l'acoustique est l'augmentation considérable des niveaux de pression acoustique en champ lointain : il y a 26 décibels d'écart entre les cas extrêmes.

\section{REFERENCES}

[1] H. Aref : Integral, chaotic \& turbulent vortex motion in two dimensional flow. Ann. Review Flud. Mech., 1983, pp. 345-389.

[2] A. Powell : Theory of vortex sound, J. Acous. Soc. Am., Vol. 36, n 1, pp. 177-195, 1964. 